Sociologie et sociétés

\title{
L’interférence parentalité-travail chez les chercheurs en post-doctorat
}

\author{
Le cas des chargés de recherches du Fonds national de la \\ recherche scientifique en Belgique
}

\section{Interferences between parenting and work among post-doctoral researchers

\author{
The case of research fellows at the Fonds national de la \\ recherche scientifique in Belgium
}

\section{Pascal Barbier et Bernard Fusulier}

Volume 47, numéro 1, printemps 2015

URI : https://id.erudit.org/iderudit/1034425ar

DOI : https://doi.org/10.7202/1034425ar

Aller au sommaire du numéro

Éditeur(s)

Les Presses de l’Université de Montréal

ISSN

0038-030X (imprimé)

1492-1375 (numérique)

Découvrir la revue

Citer cet article

Barbier, P. \& Fusulier, B. (2015). L'interférence parentalité-travail chez les chercheurs en post-doctorat : le cas des chargés de recherches du Fonds national de la recherche scientifique en Belgique. Sociologie et sociétés, 47(1), 225-248. https://doi.org/10.7202/1034425ar
Résumé de l'article

À partir de l'analyse d'entretiens menés auprès de jeunes chercheurs-parents, cet article met en avant les effets différenciés de la vie familiale sur la manière dont les individus voient et font leur travail. Il étudie le rapport au travail des chercheurs comme le produit de la rencontre entre une configuration familiale (ressources matérielles à disposition en vue de l'organisation quotidienne, attentes envers la vie familiale, la trajectoire familiale) et une configuration professionnelle (ressources matérielles et symboliques conférées par le milieu professionnel, trajectoires professionnelles observées par les individus, regard sur le métier et ses chances d'y faire carrière). En soulignant le phénomène d'interférence travail/famille, l'article invite à articuler l'analyse des interdépendances objectives entre ces deux milieux de vie à l'analyse de leurs interstructurations subjectives. 


\title{
L'interférence parentalité-travail chez les chercheurs en post-doctorat
}

\author{
Le cas des chargés de recherches du Fonds national de \\ la recherche scientifique en Belgique'
}

\section{PASCAL BARBIER}

Maître de conférences en sociologie, Université Paris 1 Panthéon Sorbonne, CESSP-CSE Courriel : pascal.barbier@univ-parisı.fr

\author{
BERNARD FUSULIER \\ Maître de recherches du FNRS et professeur, \\ Université Catholique de Louvain, GIRSEF \& \\ CIRFASE, chercheur associé au LISE/CNAM CNRS \\ IACS — Place Montesquieu i bte L2.08.04, 1348 \\ Louvain-la-Neuve, Belgique \\ Courriel: bernard.fusulier@uclouvain.be
}

\section{INTRODUCTION}

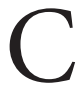
ES DEUX DERNIÈRES DÉCENNIES, l'articulation travail/famille est devenue un enjeu social et une des priorités de nombreux pays en même temps qu'elle est dorénavant reconnue comme un défi de connaissances en sociologie. Déjà, en 1977, aux États-Unis, Rosebeth Moss Kanter dévoilait des transformations sociales majeures à ce sujet, à la fois structurelles (augmentation du nombre de femmes sur le marché du travail et de familles monoparentales, tertiarisation de l'économie, importance des éléments immatériels dans le système productif, etc.) et culturelles (valorisation du développement personnel, reconnaissance d'une pluralité des styles de vie, etc.). Ce nouveau contexte, qualifié à l'époque de «postindustriel» par Daniel Bell (Bell, 1973), était propice, selon Kanter, à une redéfinition du lien entre travail et famille.

Dans l'espace sociologique francophone, si l'idée d'une imbrication de la sphère professionnelle et de la sphère familiale est avancée dès les années 1980 dans le sillage

1. Cet article résulte d'un programme de recherches sur l'interférence vie professionnelle/vie privée dans le déploiement des carrières scientifiques soutenu par le Fonds national de la recherche scientifique et dirigé par Bernard Fusulier. Nous remercions notre collègue Maria del Río Carral sans qui nous n’aurions pu bénéficier d'un matériau aussi riche à analyser. 
des études féministes (voir Collectif, 1984; Devreux, 1985), ce ne sera principalement qu'au milieu des années 1990 (Hantrais et Letablier, 1995) et surtout au début des années 2000 que la question de l'articulation sera émise (e.g. Fagnani et Letablier, 2001; Méda, 2001; Barrère-Maurisson, 2003; Fusulier, 2003; Tremblay, 2004 et 2005; Le Bihan-Youinou et Martin, 2008; Nicole-Drancourt, 2009; Pailhé et Solaz, 2009; Barrère-Maurisson et Tremblay, 2009; Périvier et Silvéra, 2010).

Ce thème est souvent couplé à l'analyse des inégalités de genre relativement à l'emploi mais aussi au rôle de l'entreprise ou des politiques publiques et des contextes sociétaux. Plus récemment, une entrée par les groupes professionnels dans le champ d'études de l'articulation travail/famille s'est affirmée (voir par exemple Fusulier, 2011a; Tremblay, 2012; Fusulier et Tremblay, 2013).

Adoptant cette perspective, nous avons lancé en 2010 un programme de recherche sur l'interférence travail/famille dans le déploiement de la carrière scientifique (voir Encadré 1) en ciblant, dans un premier temps, les chargés de recherches du Fonds national de la recherche scientifique (FNRS) en Belgique. Il s'agit de chercheurs titulaires d'un doctorat sous contrat d'emploi à durée déterminée, sélectionnés par le FNRS après une compétition hautement sélective pour mener à bien une recherche sur une période initiale de trois ans pouvant être prolongée d'un an. Contrairement aux chercheurs CNRS en France, les chercheurs FNRS sont organiquement rattachés à l'une des universités belges francophones. L'intérêt de ce groupe est qu'il est à la fois facilement identifiable et de taille modeste (une centaine de mandats sont attribués annuellement), partageant un même statut qui est valorisé dans l'espace académique et scientifique belge, c'est-à-dire qu'il constitue un vivier privilégié pour la sélection ultérieure aux postes définitifs. Il couvre l'ensemble des disciplines scientifiques. En outre, ces chercheurs sont en grande majorité trentenaires ou proches des trente ans (seules quelques personnes ont plus de quarante ans). Ils occupent donc une position dans le parcours de vie où des choix deviennent décisifs tant sur le plan professionnel que sur les plans conjugal et parental, en particulier pour les femmes qui peuvent vivre une désynchronisation entre l' «horloge biologique» et l'«horloge professionnelle» (Etzkowitz et al., 2000).

\section{Encadré 1 : Base empirique et méthodologie}

Les résultats présentés dans cet article, bien qu'inédits, doivent être resitués dans la continuité de publications (en particulier Fusulier et del Rio Carral, 2012; del Rio Carral et Fusulier, 2013) issues d'un programme de recherche entamé en 2010. Ce programme s'est déroulé en plusieurs moments et selon plusieurs méthodes d'enquête.

Dans une phase exploratoire, en 2010-2011, une étude a été réalisée par questionnaires. Cent quatre-vingt-quatre chargés de recherches FNRS appartenant à l'ensemble des champs disciplinaires universitaires (représentatifs d'une population totale de trois cent cinq individus) ont répondu à des questions portant sur leur situation professionnelle, l'organisation de leur travail, leurs pratiques de travail, leur conception de l'engagement professionnel, leur situation privée et familiale. À 
la suite de ce questionnaire, dix-huit chercheurs ont été rencontrés dans le cadre d'un entretien semi-directif. Dans un second temps, sur la base de ce travail exploratoire, une nouvelle enquête par questionnaire a été menée durant l'année académique 2011-2012 auprès des chargés de recherche en première année de mandat ${ }^{2}$. Parmi ces individus, trente ont été sollicités ( 14 femmes et 16 hommes) afin de réaliser un entretien chaque année pendant les trois ans de leur mandat postdoctoral. Ces entretiens portaient sur les trajectoires sociales et professionnelles des chercheurs, sur l'organisation de leur vie quotidienne, sur leur rapport au travail et sur leurs projets. Entre décembre 2013 et janvier 2014, à des fins comparatives, seize chercheurs supplémentaires dans leur troisième année de mandat ont participé à cette enquête (onze femmes et cinq hommes).

Dans cet article, notre matériau de base est composé de trente-deux entretiens effectués avec les chercheurs-parents (18 mères et 14 pères) tirés du corpus susmentionné, que nous complétons de quelques résultats acquis, notamment statistiques, de l'analyse de l'ensemble des enquêtes. L'objectif est de repérer la façon dont les dimensions privées et familiales affectent l'expérience du travail, alors que cette interférence reste encore peu documentée en ce qui concerne les carrières scientifiques.

Sur la base de récits compréhensifs synthétiques élaborés depuis les entretiens relatant les expériences et trajectoires des chercheurs-parents, nous avons procédé par comparaison en portant une attention aigüe à la manière dont les chercheurs voient et font leur travail, à leurs attentes envers la parentalité, à leurs conditions socioéconomiques et matérielles d'existence, à leurs trajectoires sociale et professionnelle. Cela nous a permis de dégager des logiques d'interférence qui ne sauraient être assimilées à des classes regroupant tous les chercheurs rencontrés: certains chercheurs correspondent de manière très fidèle à une logique, d'autres la partagent partiellement ou encore, d'autres s'en éloignent car ils expriment une autre logique d'interférence.

En portant son attention sur les chercheurs-parents devant faire face à une responsabilité familiale dans un contexte professionnel valorisant un engagement élevé dans le travail, cet article contribue plus généralement à souligner combien et comment la configuration familiale constitue une variable participant à la structuration du rapport au travail des individus.

Dans un premier temps, nous expliquerons pourquoi les chercheurs-parents en cours d'insertion dans la carrière scientifique représentent un sujet d'étude suggestif, voire emblématique. Nous exposerons ensuite la manière dont se joue la relation travail/ famille, et la parentalité en particulier, à travers trois logiques d'interférence dégagées de l'analyse des entretiens avec les chercheurs-parents. Enfin, nous reviendrons sur deux propriétés sociales (genre et trajectoire sociale) propices au déploiement d'une interférence perturbatrice.

2. Quatre-vingt-six questionnaires ont été recueillis, dont soixante-douze ont été entièrement remplis (41 femmes et 31 hommes), sur un total de quatre-vingt-dix-neuf individus en première année. 


\section{UN CAS D'ÉTUDE: LES CHERCHEURS-PARENTS ENTRE DEUX «INSTITUTIONS GOURMANDES 》}

La situation des chercheurs est un analyseur de l'importance de la relation travail/ famille du fait du contenu de leur travail, de certains aspects de l'organisation du milieu scientifique (plus saillants dans certaines disciplines que d'autres) et de leurs caractéristiques sociodémographiques. Ensemble, ces caractéristiques génèrent une «haute tension» dans la conduite simultanée d'une carrière professionnelle et d'une carrière familiale (Fusulier et Del Rio Carral, 2012).

\subsection{Le poids de la figure idéale du chercheur engagé et passionné}

Le milieu scientifique érige en idéal un engagement au travail entier et véhicule un ethos professionnel masculin (Beaufays et Krais, 2005; Zarca, 2006; Dany, Louvel et Valette, 2011), valorisant un surinvestissement au travail accompagné, comme on l'observe dans d'autres activités, d'un sous-investissement familial (Lapeyre, 2004; 2008). Cet aspect explique en partie pourquoi les femmes qui représentent une part importante des effectifs étudiants et doctorants depuis les années 1980 accèdent moins que les hommes aux positions académiques et scientifiques les plus valorisées et font l'objet d'une "évaporation" à travers un «tuyau percé» (Meulders et al., 2012; ANEF, 2014; SHE Figures, 2013). Le travail de recherche suppose une implication substantielle qui connaît peu la séparation entre les sphères privée et professionnelle (Del Rio Carral et Fusulier, 2013). Il faut "donner de sa personne», en consentant à des débordements d'horaires, des heures passées à travailler à domicile, la semaine et aussi le week-end, dans des tâches variées et fragmentées, aux temporalités souvent différentes qui produisent une charge mentale importante (Ait et Rouch, 2013; Datchary, 2008). De fait, parmi les chargés de recherche en première année de mandat ayant répondu à notre questionnaire en 2011-2012, seuls $10 \%$ ne travaillent que «rarement» les week-ends et $65 \%$ ne parviennent pas à complètement déconnecter de leur travail quand ils sont en congé. En moyenne, ils déclarent travailler 49 heures par semaine.

Au-delà des appartenances disciplinaires (voir Encadré 2), l'exercice idéal du métier véhiculé dans le cadre d'une socialisation professionnelle est celui d'un engagement total dans ce que Lewis Coser nomme une «institution gourmande», c'est-àdire une sphère de la vie sociale qui « demande à ses membres une disponibilité totale [...] et tente d'impliquer en son sein toute leur personnalité» (1974:4)3. On repère aisément cet idéal du chercheur tel un «lonely hero » (Benschop et Brouns, 2003) dans les récits sur l'expérience du milieu scientifique (Balibar, 2014) mais aussi dans les entretiens réalisés dans le cadre de notre enquête. Le chercheur totalement engagé et passionné qui s'investit sans compter dans la recherche et répond à toutes les sollicitations (mobilité internationale et nationale, enseignements, publications, etc.) est un étalon auquel tous les chercheurs interviewés se mesurent, que ce soit pour démontrer

3. Traduit par nos soins. C'est sous ce terme d'institution gourmande que l'université est appréhendée dans plusieurs recherches. Voir par exemple Currie et al. (2000), Hendrickson et al. (2011). 
la proximité de leur propre pratique ou, au contraire, pour marquer une distance. Le fort investissement est aussi lu comme une nécessité pour atteindre des critères d'excellence scientifique régissant les carrières professionnelles et pour maintenir sa valeur dans un milieu très compétitif ${ }^{4}$.

\section{Encadré 2: La question du champ disciplinaire et de la diversité interne au groupe étudié}

Initialement, nous avions considéré que la discipline était une variable discriminante au sein de ce groupe de chercheurs aux côtés du genre, de l'âge et de l'origine sociale. À l'épreuve de nos analyses, il s'avère en effet qu'elle a un impact sur le rapport au salaire (moins valorisé dans les sciences exactes), sur le pouvoir de marché anticipé (la capacité de se reconvertir en dehors du monde académique étant, par exemple, moins importante dans les sciences humaines et sociales) et sur la norme du nombre de publications annuelles (plus faible dans les sciences de la santé). Pourtant, lorsque l'on analyse les rapports subjectifs à la carrière scientifique, le champ disciplinaire n'intervient pas directement (Fusulier et del Rio Carral, 2012). Évidemment, les contraintes organisationnelles varient entre un archéologue qui doit planifier des séjours de longue durée sur un terrain de fouille, un biologiste qui met en culture des cellules vivantes ou un historien qui travaille sur des archives. De telles variations se retrouvent cependant à l'intérieur même d'une discipline (entre un sociologue réalisant une ethnographie et un sociologue opérant par analyse secondaire de bases de données, par exemple). Il existe également une diversité de sous-cultures de centre, de laboratoire, voire d'université qui produisent des conventions organisationnelles et caractérisent les relations sociales de travail (entre pairs, entre seniors et juniors, entre académiques et administratifs...). La morphologie sociodémographique des champs disciplinaires, des secteurs, des centres... est tout aussi plurielle. Cette grande diversité participe de ce que nous appelons les configurations professionnelles, lesquelles n'excluent pas pour autant l'existence d'un «ethos professionnel générique» (Fusulier, 2011b) et d'une régulation transversale aux disciplines (et aux institutions). La spécification de ces configurations et de leurs effets sur les carrières relève d'une recherche en soi, recherche engagée depuis janvier 2014, dans le cadre d'un projet européen (GARCIA) portant sur les similarités et différences dans les trajectoires scientifiques selon le genre de chercheurs appartenant, d'une part, au secteur des sciences et technologies et, d'autre part, au secteur des sciences humaines et sociales.

4. L'accès à un poste stable dans la recherche scientifique engage une compétition entre un nombre élevé de candidats (belges et étrangers) pour un nombre réduit de postes définitifs. Il n'existe pas d'estimation fiable du bassin de concurrence, mais nous pouvons observer que le FNRS a financé ces cinq dernières années (de 2009-2010 à 2013-2014) 499 nouveaux mandats de chargés de recherches pour 98 nouveaux mandats de chercheurs qualifiés (définitifs), soit un ratio de $20 \%$ sachant que le recrutement au poste de chercheurs qualifiés est ouvert à tous les docteurs, belges ou étrangers, qu'ils aient été chargés de recherches au préalable ou non. 


\subsection{Une organisation du travail compatible avec un engagement total}

La «gourmandise» du milieu de la recherche scientifique envers l'investissement de ses membres satisfait, chez les chercheurs, une demande de travail potentiellement infinie. Parce que le travail ou une partie de celui-ci (selon les disciplines et l'objet des recherches) peut se faire à tout moment et en tout lieu (ne fût-ce que pour lire un texte, rédiger un article, analyser des statistiques, répondre aux courriers électroniques...), parce que les chercheurs se disent le plus souvent libres de chercher et de s'organiser $^{5}$, la compétition nourrit une course au travail qui ignore les contraintes extra-professionnelles et installe le travail au cœur de la vie privée. L'autonomie rend virtuellement possible la réalisation d'un idéal d'engagement total dans la carrière scientifique. Et comme cet engagement ne garantit jamais d'obtenir un poste définitif de scientifique ou d'académique, la liberté de chercher et l'autonomie de s'organiser contribuent ensemble à la production d'une forme de "servitude volontaire», associée à une responsabilisation des succès comme des échecs qui caractérise tout un pan du monde du travail contemporain (Sarfati, 2012).

L'enquête quantitative exploratoire montre que cette «servitude volontaire» et cette responsabilisation sont soutenues par une forte satisfaction tirée de l'exercice du travail: $87 \%$ déclarent que le travail scientifique leur procure une grande satisfaction et plus de $80 \%$ que leur activité professionnelle occupe une grande place dans leur existence. Et de fait, neuf chercheurs sur dix espèrent, en premier lieu, obtenir un poste permanent dans la recherche universitaire, tout en sachant que la probabilité de l'obtenir est relativement faible (Fusulier et del Rio Carral, 2012). Cette satisfaction est directement apparue dans les entretiens avec des chercheurs décrivant souvent la recherche comme une source de plaisir quotidien, la quête de la «vérité» étant assimilée à un jeu, à une création ou à la participation à un «bien commun».

\subsection{Une étape importante dans le parcours professionnel et le parcours de vie}

Les chercheurs étudiés sont à un moment charnière de leur trajectoire professionnelle et personnelle. En effet, l'obtention d'un mandat postdoctoral du FNRS, qui intervient parfois au terme d'une séquence préalable d'accumulation de contrats postdoctoraux, constitue l'une des dernières étapes dans la compétition pour accéder à un poste stable. En dehors des postes définitifs de chercheurs qualifiés FNRS ou d'académiques, les opportunités de poursuivre son parcours dans la carrière scientifique universitaire se raréfient. Il existe donc chez eux une tension pouvant les stimuler à intensifier ou, à l'inverse, à affaiblir leur engagement dans le travail. Ils sont à une étape de leur trajectoire professionnelle où se révèlent de manière potentiellement dramatique (sous la forme de l'expérience d'un vide professionnel) la rentabilité des efforts consentis depuis le début de la thèse.

5. Par exemple, plus de $90 \%$ des répondants à notre enquête auprès des chercheurs en première année de mandat déclarent qu'ils sont plutôt d'accord ou tout à fait d'accord avec la proposition: «Je suis entièrement libre d'organiser mon horaire de travail comme je le veux » et ils sont plus de $75 \%$ à avoir cette opinion en ce qui concerne le lieu de travail. 
De plus, les chercheurs-parents sont à un moment singulier de leur parcours de vie: ils sont jeunes parents et considèrent souvent nécessaire, les hommes comme les femmes, de consolider les fondations de cette parentalité. Ils entendent donner du temps à leur famille, et surtout accorder à leurs enfants du «quality time» (Hochschild, 1997).

C'est donc en raison du contenu de leur travail (intense, relativement flexible, débordant), de ses exigences et modalités d'exécution (emploi précaire, concurrence, mobilité géographique, travail à domicile), de l'idéal d'engagement professionnel attendu par leur milieu professionnel et du positionnement dans leurs parcours de vie et professionnel que les jeunes chercheurs sont en mesure de révéler avec force les effets d'une interférence travail/famille, en particulier sous l'angle de la relation entre la parentalité et le rapport au travail.

\section{TROIS LOGIQUES D'INTERFÉRENCE: RENFORCEMENT, APAISEMENT, RECADRAGE}

L'influence de la vie familiale sur la manière dont les chercheurs-parents interviewés voient et font leur travail s'exprime de façon variable dans les discours. Trois logiques d'interférence ont été dégagées à partir de l'analyse de l'interaction entre la configuration familiale (ressources matérielles et symboliques disponibles pour organiser la vie quotidienne, attentes envers la vie familiale, trajectoire familiale) et la configuration professionnelle (ressources matérielles et symboliques conférées par le milieu professionnel, trajectoires scolaire et professionnelle, regard sur le métier et chances d'y faire carrière). Ces configurations ne sont toutefois pas traitées de façon entièrement symétrique, car nous avons privilégié la mise en évidence de la configuration familiale dans la construction du rapport au travail, pour bien montrer combien les conditions de la vie privée en sont l'une des dimensions constitutives.

\subsection{Quand parentalité et travail se renforcent (logique de renforcement)}

Une première logique consiste en un renforcement mutuel du rapport au travail et du rapport à la parentalité. Par la pression temporelle qu'elle instaure, la parentalité a transformé la forme de l'investissement au travail, les chercheurs devant notamment consentir à des départs anticipés du lieu de travail. Cependant, comme ces contraintes sont compensées par une intégration du travail dans la sphère familiale, la nature de l'engagement professionnel demeure inchangée. Ainsi, la vie familiale est non seulement jugée compatible avec une vie professionnelle intense, mais elle est surtout considérée comme favorable à son accomplissement. En effet, dans cette logique, la parentalité ressemble moins à un facteur de désordre, nuisible à la vie professionnelle, qu'à une source de structuration favorable. Les enfants structurent, imposent un rythme, procurent un sentiment de rationalisation de l'investissement au travail, autant d'aspects qui apparaissent comme un véritable gain: les chercheurs disent alors travailler plus vite et mieux que lorsqu'ils étaient sans enfant, en thèse, moment considéré comme une période où le travail occupait la majeure partie de l'existence. 
Ainsi, sous certaines conditions, la famille peut enrichir l'expérience du travail en pesant favorablement sur l'engagement des individus. Elle donne l'impression d'être plus productif et organisé, d'être plus pro, à un moment où cela est justement considéré comme nécessaire afin, selon Jacin, par exemple, chercheur en histoire, père de deux filles (deux ans et demi et six mois) et marié à une directrice d'un centre d'archives, de booster son $C V$. Le plaisir tiré du travail est même décuplé par les possibilités qu'il offre pour exercer une parentalité épanouie à travers notamment une capacité de dégager du temps le moment opportun.

\subsubsection{Des appuis configurationnels forts}

Cette logique exprime un processus de renforcement mutuel entre parentalité et rapport au travail qui contribue à produire un sentiment d'accomplissement dans les deux sphères. Elle résulte toutefois de la rencontre de configurations professionnelle et familiale singulières, livrant des appuis favorables. Il en va ainsi de la possibilité d'assurer une prise en charge partagée des enfants entre le chercheur, le conjoint, disponible le matin et/ou le soir à horaires fixes, ou encore des grands-parents résidant à proximité. Cette configuration permet de rester sur le lieu de travail de longues heures (le soir notamment) mais aussi de faire face aux longues absences pour des séjours scientifiques à l'étranger. Un lieu de résidence proche du travail, de l'école ou de la crèche renforce les latitudes organisationnelles octroyées par l'entourage familial. La mutualisation des obligations parentales entre parents et grands-parents suppose une organisation domestique finement gérée à la journée, à la semaine et même au mois.

Cette économie domestique peut s'enraciner dans un couple ancien ayant traversé l'expérience de la thèse, avec une forte convergence de vues, cette dernière permettant au conjoint de considérer comme normales les épreuves qui composent l'accès à un emploi stable dans la recherche scientifique et donc de s'y plier. Le conjoint peut, par exemple, prendre en charge les coûts organisationnels des séjours à l'étranger, parce qu'il comprend les enjeux du métier (notamment la nécessité de s'y engager) ou parce que pour lui aussi la mobilité constitue une exigence professionnelle appréciée. C'est par exemple le cas d'Alexis, chimiste âgé de 32 ans et père de deux garçons (2 et 6 ans), dont la femme manager, d'origine irlandaise, se dit prête à se déplacer en Europe au nom d'un goût pour la mobilité. La convergence de vues au sein du couple se traduit aussi par une organisation concertée des évènements quotidiens et professionnels (la carrière de l'un étant mise entre parenthèse pour celle de l'autre, et vice-versa). En plus de renforcer la compréhension des enjeux liés à la carrière scientifique, une certaine homogamie, voire endogamie, permet au chercheur de travailler le soir ou le week-end, en même temps que son conjoint (cela est parfois décidé par mail ou téléphone comme pour Sybille, chercheure en biologie dont le conjoint, informaticien, est amené à travailler à son domicile). De fait, l'image du chercheur entièrement engagé dans son travail est non seulement valorisée mais joue comme une référence à incarner. Un tel engagement n'est pas sans lien avec le plaisir 
éprouvé dans l'exercice du travail. Plusieurs chercheurs qui soutiennent cette logique lisent leur travail sur le registre de la passion, laquelle est vue comme une condition de réussite dans la concurrence pour les postes définitifs. La motivation est entretenue par divers indicateurs (des prix, des bourses, des articles dans des revues prestigieuses, des invitations pour des séjours scientifiques à l'étranger...) et un sentiment de reconnaissance éprouvé auprès de collègues et de la «communauté scientifique». Une telle reconnaissance engendre un solide sentiment de confiance et une volonté chez le chercheur de faire corps avec le milieu scientifique en s'engageant fortement dans son travail.

\subsubsection{Des appuis qui ne sont pas sans liens avec des conditions plus structurelles}

La rencontre entre des facilités organisationnelles et une volonté d'engagement dans le travail, soutenue par des signes de réussite, transforme donc l'entrée dans la parentalité en un évènement vertueux du point de vue du travail. Le travail envahit la vie privée, sans que cela n'apparaisse comme un coût grâce à un soutien sans faille de l'entourage familial et à la foi dans les qualités du milieu scientifique. La parentalité offre un gain en termes d'organisation jugé profitable au travail. Cette logique dépend toutefois de ressources matérielles et symboliques conjoncturelles et structurelles. Il en est ainsi des équipements présents sur le territoire (une crèche, une ligne de bus, une autoroute, etc.) dont le bénéfice peut dépendre de facilités économiques en partie offertes par l'entourage (une avance sur héritage, un apport financier dans le cadre d'un prêt, etc.). De la même manière, la confiance dans les vertus du milieu et en ses qualités professionnelles est le fruit d'une socialisation réussie, d'une familiarité, dès le début de Master, avec le milieu scientifique permettant de faire les bons choix (d'articles, de jury de thèse, etc.) à l'image de Jacin, dont le père est lui-même chercheur. Autrement dit, ces ressources sont aussi structurellement distribuées puisqu'elles ne sont pas sans lien avec l'origine sociale. Des chercheurs issus de milieux populaires $^{6}$ démontrent inversement une faible confiance en eux, une tendance au retrait face à la concurrence, comme l'exprime Manuella, mère de deux enfants ( 3 ans et 1 an) en couple avec un architecte et fille de carreleur qui insiste sur ses difficultés pour prendre confiance en elle et faire front dans un milieu où il ne faut pas «avoir peur de laisser des plumes». Enfin, on le verra, l'éventualité d'éprouver cette forme de renchérissement de l'expérience du travail du fait de l'arrivée d'un enfant pose inévitablement la question du genre, sans qu'elle soit pour autant totalement déterminée par lui.

6. L'enquête exploratoire a révélé une nette surreprésentation, chez les chercheurs, d'individus ayant des parents diplômés de l'enseignement supérieur ou universitaire ( $71 \%$ des pères des chercheurs et $66 \%$ des mères des chercheurs); $80 \%$ des chercheurs se sentent issus d'un milieu socioéconomique "plutôt favorisé» ou «favorisé». 


\subsection{Quand la parentalité apaise et libère des inquiétudes de la carrière (logique d'apaisement)}

La naissance et la présence d'un enfant peuvent également agir de manière vertueuse non pas sous la forme d'un renforcement mais sous celle d'une compensation du délitement du sens accordé au travail. Sur un terrain fragilisé, la parentalité octroie un sentiment élevé de normalité et parfois le sentiment de recouvrer une lucidité. Avec la parentalité, l'activité professionnelle est mise au pas, contrainte de s'ajuster aux exigences jugées prioritaires de la famille. Cela se traduit par un glissement des aspirations personnelles du travail vers le domaine familial en raison, moins d'une désaffection envers le contenu du travail, le plaisir tiré de l'exécution du travail demeurant intact, que d'un rejet des conditions nécessaires à l'obtention d'un statut stable. Dès lors, la famille donne un sens à une mise à distance des exigences professionnelles. Elle permet légitimement d'en faire moins, de seulement profiter de l'exercice d'un travail toujours vécu comme plaisant et d'éprouver ce qui est perçu comme une «normalité». Les chercheurs exprimant cette logique mettent en avant leur réussite familiale, la qualité de vie face à ce qu'ils jugent comme du "gâchis", celui des autres, qui risquent de «se retrouver sans rien» à l'issue d'une compétition qu'ils estiment injuste et rarement payante. La famille apaise donc ces inquiétudes professionnelles et délivre une prise de conscience relativement à la contingence du milieu scientifique. Ces chercheurs sont les plus critiques envers les recrutements et les critères de sélection qui feraient la part belle au «copinage» et attendraient des candidats une capacité de faire «tous les sacrifices». Ainsi, en insistant sur leur «normalité», ils dénoncent l'anormalité d'un milieu qui méconnaît les contraintes ordinaires de l'existence.

\subsubsection{Une distanciation déjà présente à l'égard de la carrière...}

Chez ces chercheurs, la parentalité intervient au bout d'une trajectoire de distanciation envers la carrière et durcit une remise en question du sens accordé au travail et surtout à la situation professionnelle. La foi dans le milieu scientifique que certains déclarent avoir eu chevillée au corps s'est progressivement effacée à travers des évènements défavorables compliquant la poursuite d'une carrière comme une sortie de thèse difficile. Par exemple, la période d'après thèse a contraint Sarah, biologiste âgée de 33 ans, mariée à un chercheur et mère d'une fille, à se tourner une première fois vers une recherche d'emploi dans le privé, avant d'obtenir un postdoc qu'elle qualifie d' «alimentaire», puis une série de petits contrats entrecoupés de périodes de chômage. Il peut également s'agir de candidatures sans succès, de désaccords avec le directeur de recherche, de conflits avec les collègues dans le cadre de relations de travail qui se transforment en une compétition interpersonnelle dans laquelle certains refusent de s'engager ${ }^{7}$.

7. D'après l'enquête menée en 2010-2011, un chercheur sur deux considère que l'importance de la concurrence entre jeunes chercheurs dans le champ scientifique constitue un frein dans sa volonté de poursuivre une carrière scientifique. 


\subsection{2 ...compensée par la parentalité}

Face à ces évènements, l'arrivée des enfants confirme, voire légitime, un retrait partiel. Elle accélère ce processus de distanciation en facilitant une «déconnection» avec le travail jugée nécessaire lorsque les autres chercheurs seraient «toujours connectés». C'est tout l'intérêt des enfants pour ces chercheurs qui se réjouissent d'en revenir à des aspects de leur existence jugés "plus essentiels», "plus importants». La parentalité semble remplir un espace libéré par la vie professionnelle et, contrairement à l'incertitude qui caractérise l'engagement dans une carrière sans promesse, offrir des certitudes sur le long terme. Miriam se réjouit ainsi de sa seconde maternité, longuement désirée au moment où, à ses yeux, ses perspectives professionnelles se ferment et qu'elle se plaint d'un manque de soutien de la part de son directeur de recherche. Cette chercheure en astrophysique âgée de 35 ans, qui a longtemps fait les efforts de la mobilité et de la productivité, se réjouit aujourd'hui de ne rien regretter, parce qu'elle a joué le jeu aussi longtemps qu'il le fallait à ses yeux (selon elle, c'est aujourd'hui «le système» qui est responsable de sa situation, et non son comportement). Et au-delà de cette réjouissance prenant la forme d'une extériorisation de l'échec, Miriam insiste sur ce qu'il lui restera, des gratifications privées, à l'inverse, dit-elle, d'autres chercheurs qui ont tout donné à la recherche.

Je me dis voilà ça marche, tant mieux, ça marche pas, je sais que je ne suis pas la seule dans le cas et il faut vivre avec sinon on ne pense plus qu'à ça quoi. Donc j'ai mes moments de crise mais je suis contente d'y être passée quoi. Parce que je sais que certaines... C'est aussi d'avoir vu comment d'autres postdocs qui apparemment ne voudraient que faire ça, ça, ça. Et je me dis: «Mais ils vont être déçus à un moment donné quoi. Et si vraiment ils ne vivent que pour ça et s'ils ne pensent qu'à ça ils se gâchent la vie quoi.» [Miriam, 35 ans, astrophysicienne, deux enfants âgés de 2 et 7 ans, mari fonctionnaire]

Ces certitudes sont vécues comme protectrices: la parentalité contraindrait à se défaire d'une forme de "naïveté», à réfléchir à des stratégies de reconversion ou à une réévaluation de l'engagement au travail, plus «stratégique», dans des actes potentiellement utiles, comme des enseignements afin d'obtenir un poste de professeur. C'est par exemple la «stratégie» de Jonathan, chercheur en mécanique, père de trois enfants et marié à une psychologue, qui ne s'investit aujourd'hui que dans les tâches lui permettant de se positionner sur des postes d'enseignants dans son université.

En somme, la parentalité apparaît chez ces chercheurs comme une transition vertueuse vers un ailleurs professionnel anticipé. Cette logique qui suppose une qualité et une stabilité de la sphère privée capable d'alimenter l'apaisement à l'égard du travail et de l'emploi - stabilité et qualité dont Arlie Hochschild a montré qu'elles ne constituaient pas une évidence (1997) — permet de prendre ses distances avec l'incertitude qui caractérise l'insertion dans la recherche scientifique. Dans le même temps, elle est brandie comme une justification permettant de plus facilement se distancer d'un travail chronophage, d'une régulation des carrières jugée destructrice. Même si le jeu continue d'être joué, avec une plus grande stratégie, chaque investissement étant compté à la hauteur des gains potentiels, un deuil d'une carrière universitaire s'opère. 
Aux yeux de ces chercheurs, tous les efforts ne suffiraient pas à garantir une position stable car même les chercheurs les plus engagés ne sont pas assurés de succès. Autrement dit, s'ils venaient à échouer, ils perdraient sur tous les tableaux lorsque les chercheurs porteurs de cette logique seraient gagnants du point de vue de leur vie privée.

\subsection{Quand la parentalité recadre sous contrainte l'investissement professionnel (logique de recadrage)}

Un troisième type d'interférence observé chez les chercheurs-parents consiste en l'expérience d'un recadrage de l'investissement professionnel par les contraintes de la parentalité. Tout en modérant une ardeur au travail considérée comme débordante, ce recadrage produit une mise en tension d'un rapport au travail très positif. Pour Amandine, chercheure en sociologie, âgée de 39 ans, en couple avec un professeur des écoles et mère de deux enfants ( 3 et 7 ans), «ce n'est pas le métier qui est un problème, mais le fait d'être une mère».

\subsubsection{Le vécu d'un empêchement...}

Tout en accomplissant un projet familial ancien et jugé hautement satisfaisant, ces chercheurs entendent aussi «faire ce qu'il faut» pour le travail. Et à ce titre, les enfants apparaissent comme une contrainte avec laquelle il faut composer puisqu'il «faut bien» aller les chercher, il «faut bien» s'en occuper. Leur volonté de s'engager dans le travail tient au plaisir retiré de l'exercice du travail qui selon Kat, chercheure en littérature et mère de deux enfants, est un «loisir, une satisfaction, un épanouissement». Il tient également à un parcours lu par soi et autrui comme prometteur: un classement élevé au sein des promotions d'étudiants lors de la formation universitaire, lors des délibérations attribuant les mandats d'aspirant du FNRS (mandats permettant de réaliser une thèse en quatre ans) ou de chargé de recherche du FNRS, etc. Ce début de carrière prometteur apparaît contrarié par un événement (une grossesse, un changement de spécialité, un échec dans une recherche, une maladie...) produisant le sentiment d'être entravé. Jessica, psychologue et mère de deux enfants, en couple avec un consultant, estime par exemple avoir perdu beaucoup de temps lors de son congé maternité et des mois qui suivirent. De la même manière, Séverine, biochimiste âgée de 31 ans, mère d'une fille âgée de 10 mois et en couple avec un commercial exprime des difficultés importantes lors des mois précédents et suivants son accouchement durant lesquels: «Je ne savais pas vraiment ce que je faisais. Je lisais des articles, j'essayais de faire deux-trois manipes mais vraiment pour dire que $j$ 'avais fait quelque chose. »

Alors que le mandat de chargé de recherche apparaît comme une période de l'insertion professionnelle charnière exigeant un travail intensif, leur disponibilité pratique et subjective diminue. Ils en sont à la dernière étape de la compétition, et le jeu semble en valoir la chandelle, notamment depuis qu'ils ont fait l'expérience d'un poste qui ressemble fortement à celui qu'ils pourraient obtenir sous un statut stable (une «fenêtre sur le paradis» pour Sonia, mère de deux enfants et en couple avec un 
chercheur). Le quotidien de ces chercheurs repose donc sur l'expérience d'une urgence dans le travail emmêlée dans un sentiment d'empêchement par la famille. Si ce vécu est fortement manifesté par des mères, il ne leur est pas propre. Laurent, chercheur en sciences humaines, âgé de 33 ans, père d'un fils âgé de quelques mois et en couple avec une accessoiriste formule des inquiétudes sur sa capacité de faire front. Très concerné par l'éducation de son fils, il veille à être présent à ses côtés, à participer avec sa conjointe aux soins portés à son fils. Cela le contraint à ne pas faire ce qu'il faut au travail, alors qu'il s'estime dans une situation cruciale du point de vue de sa carrière.

\subsection{2 ...produit par des défaillances dans la configuration familiale}

Les frustrations sont décuplées par une défaillance dans la configuration familiale. L'absence d'une ressource essentielle, même compensée, en théorie, par la présence d'autres ressources organisationnelles ou par la compréhension des collègues et de la hiérarchie relativement aux obligations parentales ${ }^{8}$, alourdit le coût d'un accès à la carrière scientifique ardemment souhaité. Il peut s'agir d'une localisation géographique défavorable de la crèche, d'un long trajet domicile-travail, d'un conjoint indisponible du fait de son travail (commercial et souvent parti à l'étranger pour Séverine) ou du fait d'une vision traditionnelle du partage du travail domestique et parental chez Blandine, chercheure en biologie dont le conjoint est vétérinaire indépendant. Cela peut également venir d'enfants à la santé fragile nécessitant une prise en charge régulière ou encore d'un isolement vis-à-vis de l'entourage familial. En conséquence, la vie de famille alourdit l'exercice du travail: les journées sont raccourcies et la configuration familiale se prête peu à une reprise du travail en fin de journée, car le conjoint ne travaille pas le soir, car les charges domestiques sont trop importantes, etc. Or, il apparaît pourtant nécessaire à ces chercheurs de «faire des heures» et de "produire des résultats".

Ces chercheurs s'emploient, dès que possible, à "gagner» du temps dans les interstices de la vie familiale au détriment, le plus souvent, de la vie conjugale ou en se libérant des contraintes parentales: lorsque la crèche retarde d'une heure la fin de la journée de prise en charge, lorsque leurs parents se libèrent pour garder leurs petitsenfants ou lorsque leurs enfants grandissent et font «leurs nuits»... Ils s’imposent alors une nouvelle efficacité, un engagement supérieur dans le travail afin d'« en profiter» et d'écrire des articles. Mais en dépit de ces efforts, le volume de travail qu'ils effectuent ne les satisfait pas car les «vrais chercheurs» restent au laboratoire pendant de longues soirées. Ainsi, lorsque la comparaison avec l'idéal d'investissement professionnel du chercheur engagé procure un sentiment valorisant de «normalité» chez ceux qui ont fait le deuil de leurs ambitions professionnelles (logique d'apaisement), l'effet est ici tout autre. Les chercheurs ressentent une culpabilité envers ce qu'ils assi-

8. Compréhension qui s'incarne parfois par des aides ponctuelles, notamment dans le cas de recherches sur des matières vivantes où des collègues terminent certaines manipulations se déroulant audelà de $19 \mathrm{~h}$. 
milent à un défaut professionnel. Ainsi, en dépit du soutien dont elle dit bénéficier de la part de son chef, jeune père, Séverine ressent tout de même une forte contrainte:

On a des thésiens [doctorants] qui sont là et ils travaillent toute la journée de 9 à 18. [...] Et donc à $17 \mathrm{~h} 15$ on est en train de regarder l'heure parce que dans cinq minutes il faut absolument qu'on parte parce que sinon on sera en retard à la crèche. [...] c'est vrai que le regard de la personne qui n'a pas d'enfants n'est pas évident quoi. [Séverine, 31 ans, biochimiste, une fille âgée de 10 mois, conjoint commercial]

Cette frustration qui émerge avec le recadrage du travail par la vie familiale ne touche pas directement le plaisir tiré de l'exercice du travail, toujours puissant. Il porte sur le sens conféré à l'engagement. Alors que ce sens était solide et structurant, fondé sur des indices de réussite professionnelle et sur le partage d'un illusio du champ scientifique rendant légitime les efforts attendus, il est remis en cause par l'augmentation du coût d'accès à la carrière scientifique imposée par la vie familiale (coût en énergie, en frustration, en sentiment de culpabilité d'en demander beaucoup à son entourage et de ne pas être à la hauteur des exigences du milieu). Les efforts consentis se transforment alors en "sacrifices», terme très présent dans les récits. Relativement à ses nécessités professionnelles et ses contraintes familiales, Séverine exprime le regret de ne plus avoir de temps pour elle, pour son travail, pour sa fille et, finalement, de s'imposer une rationalisation où tout «doit devenir efficace».

\subsubsection{Une double culpabilité: ni le bon chercheur ni le bon parent (la bonne mère)}

Certains chercheurs expriment de l'agacement envers un quotidien «trop » routinisé et des moments passés avec leurs enfants soumis à l'exigence de l'efficacité. Du fait de la prégnance d'un idéal parental structurant, forgé par une forte perméabilité aux normes sociales de parentalité, cet effet perturbateur de la vie familiale sur l'appréciation du travail peut s'accompagner d'une interrogation sur la capacité d'être un «bon parent». Par exemple, Blandine exprime la même insatisfaction générale, dans son travail envers lequel elle estime en faire trop peu et envers ses enfants, avec lesquels elle pense être parfois trop dirigiste, ce qu'elle juge contraire à l'épanouissement familial. Les normes sociales de la parentalité contemporaine (axées sur le bien-être de l'enfant) façonnent ainsi un point de référence dans la manière d'être parent qui s'ajoute à un point de référence dans la manière d'être un «bon professionnel». Ce référentiel du «bon parent» se nourrit des influences du milieu professionnel qui actualise un ensemble de représentations sur la parentalité, des interactions avec les parents d'élèves à l'école, des professionnels de l'éducation à la crèche qui désapprouvent de voir l'enfant récupéré tard le soir, ou de l'entourage amical et familial qui s'interroge sur le sens du temps passé au travail, sur les sacrifices consentis, le plus souvent reliés à la vie des enfants... Ce faisceau d'influences fabrique des attentes familiales élevées ( $\mathrm{du}$ point de vue de la qualité de vie des enfants) cumulées à des attentes professionnelles.

On mesure avec clarté les contours de ce tiraillement chez Manuella (anthropologue, deux enfants âgés de un et trois ans, conjoint architecte). Cette dernière exprime une double culpabilité de ne pas suffisamment s'investir auprès de ses enfants et de 
son travail, l'allocation en énergie et en temps dans une sphère nourrissant une culpabilité de délaisser l'autre sphère. Elle se sent coupable de ne pas être à la hauteur des exigences du métier lorsque, pour garder son enfant, elle reste travailler à domicile, là où elle travaille moins bien à ses yeux. Certes, cela est une «chance incroyable» "par rapport à d'autres mamans [d']avoir cette flexibilité, c'est génial quand les enfants sont petits ». Mais cette même largesse est aussi vue comme la principale difficulté car elle dit apprécier son travail et vouloir lui accorder un investissement en mesure de lui offrir une position professionnelle stable. En effet, la flexibilité organisationnelle offerte par son travail l'oblige, selon elle, à donner le change, à se contraindre à une discipline ( «je suis tout le temps dans devoir faire quelque chose»). La vie familiale qui interrompt le travail à domicile nourrit alors un sentiment de ne pas être une chercheure telle qu'elle devrait l'être. C'est lors de ces moments passés à travailler chez elle, à côté de son fils, que Manuella regrette de ne pas s'occuper correctement de son enfant (lorsque, par exemple, elle joue «le joker de lui mettre un film» pour pouvoir travailler) tout en n'effectuant pas complètement son travail. Alors, dit-elle, «tout devient une course. [...] et je me dis que lui aussi il doit le sentir quoi. Comme s'il gênait. Parce que moi je veux travailler. Lui il est là. À un moment ça va se reporter sur lui.» Chez Manuella, cette imbrication étroite entre une culpabilité envers le travail et une culpabilité envers la famille se dessine explicitement autour de la question de l'heure de retour au domicile. En raison de la flexibilité dont elle peut jouir dans ses horaires de travail, c'est Manuella qui prend en charge le retour des enfants. Dans cet acte, se joue et se rejoue quotidiennement une tension inhérente à son expérience du travail et de la famille: quitter le travail et donc se soustraire à un environnement professionnel attendant une présence étendue, c'est s'exposer au regard de collègues qui travaillent tard et se défaire d'un idéal d'engagement professionnel structurant; retarder le départ du travail et donc laisser son fils plus longtemps à la crèche, c'est s'exposer aux remarques de l'entourage et des salariés de la crèche sur les bienfaits du temps familial. Mais lorsque son conjoint prend en charge les enfants, pour en "profiter» (terme qui dévoile la manière dont elle envisage les relations familiales), elle se régale de son travail, qu'elle estime exercer comme cela est nécessaire:

Le mercredi je peux partir super tôt pour revenir super tard parce que c'est lui qui est avec eux toute la journée. On ne doit pas les déposer à la crèche, pas les déposer à l'école. [...] Qu'ils passent du bon moment et je travaille bien. En général, c'est un jour où je travaille bien. [Manuella, anthropologue, deux enfants âgés de un et trois ans, conjoint architecte]

À travers le cas de Manuella, on constate un double recadrage qui n'est pas sans effet sur le rapport au travail. Ici réside un tiraillement issu des «injonctions contradictoires à l'exercice idéal de ce métier et de celui de mère» (Marry et Jonas, 2005: 70) qui exprime un effet réciproque entre la vie familiale et le travail.

\section{EFFETS D'INTERFÉRENCE ET PROPRIÉTÉS SOCIALES}

Les trois logiques d'interférence présentées ci-dessus peuvent être ordonnées en deux types d'effet distinct d'interférence entre vie familiale et vie professionnelle. À travers 
les logiques du renforcement et de l'apaisement se dessine un effet vertueux de la vie familiale sur l'expérience subjective du travail. À l'inverse, la vie familiale est susceptible de produire un effet perturbateur à travers un recadrage de l'investissement professionnel vécu sous le registre de l'imposition. Interférence vertueuse, interférence perturbatrice: se dévoilent ici des ressorts majeurs du rapport au travail de ces chercheurs. Ils s'élaborent dans des trajectoires de vie, des trajectoires professionnelles mais aussi dans des aspects souvent relégués dans l'analyse du travail: les conditions matérielles d'existence, les ressources organisationnelles, les trajectoires conjugales et parentales. Cependant, ils dépendent également des propriétés sociales des individus. En effet, notre enquête a permis de distinguer deux propriétés classiques en sociologie qui pèsent lourdement sur l'exposition aux effets vertueux ou perturbateur de l'interférence travail/famille: le genre et l'origine sociale. Les effets du premier sont aujourd'hui connus et documentés grâce aux travaux sur l'accès aux positions professionnelles supérieures (e.g. Blair-Loy, 2003; Marry, 2004; Buscatto et Marry, 2009; Le Feuvre et Lapeyre, 2013) et notamment à ceux sur l'enseignement (e. g. Jarty, 2009; Cacouault, 2007) et sur l'université et la recherche (e.g. Benschop et Brouns, 2003; Zarca, 2006; Musselin et Pigeyre, 2008; Pigeyre et Sabatier, 2011).

Plusieurs mécanismes sont identifiés. Ils touchent à la fois à la socialisation et à l'organisation du travail qui discriminent les carrières scientifiques des hommes et des femmes, et qui produisent le phénomène du «tuyau percé» (Alper, 1993) : par exemple, la structuration du champ scientifique autour d'un habitus masculin (Beaufays et Krais, 2005); l'effet «Matilda» pour les femmes (Rossiter, 1995) versus l'effet «Saint Mathieu» (Merton, 1968) pour les hommes; ou encore la cooptation au sein d'un «old boys'club» qui prodigue des conseils et offre des opportunités de carrière (Case et Richley, 2014). Et lorsque l'on observe le travail et les carrières scientifiques, on constate que ces mécanismes s'enracinent dans un conflit travail/famille (objectif et subjectif) davantage présent du côté des femmes (Marry et Jonas, 2004).

Prolongeant ces travaux, notre enquête apporte à cet égard une double contribution. Elle souligne d'abord l'effet d'une qualité propre au travail scientifique (même si elle est partiellement observable dans d'autres lieux du monde du travail), son autonomie et sa flexibilité, qui accroît les effets potentiels d'un rappel à l'ordre domestique pour les chercheures surtout. Elle montre ensuite que le genre et la situation familiale ne jouent pas seuls dans la fabrique des trajectoires professionnelles: ils s'imbriquent dans des trajectoires sociales pour produire des rapports au travail spécifiques.

\subsection{La confirmation d'une différence majeure entre hommes et femmes}

Si l'on observe une interférence parentalité-travail chez les hommes et les femmes, on ne l'observe pas chez les femmes comme chez les hommes9. Les hommes éprouvent

9. Ce qui, en soi, manifeste une transformation par rapport aux pères rencontrés dans les années 1980 par Michèle Ferrand qui ne déclaraient aucun effet de leur vie familiale sur leur travail (1984). Ce changement pourrait être lié à l'avènement des «nouveaux pères» (De Singly, 1996), et au fait que les 
certes eux aussi l'imposition d'une limite par la vie familiale, augmentant le coût d'accès à la carrière scientifique et altérant le sens de l'engagement dans le travail. Toutefois, cette altération ne s'accompagne pas, pour les hommes rencontrés en tout cas, d'une crainte d'imposer à la vie familiale une contrainte professionnelle. Le recadrage porte le plus souvent sur l'investissement dans le travail et s'accompagne rarement d'inquiétudes envers la parentalité (ces inquiétudes portant plutôt sur les loisirs et la vie amicale). Avec ce constat d'une différence significative dans l'appréciation des coûts de l'interférence entre travail et famille sur l'entourage familial, on retrouve chez les chercheurs un résultat important de la sociologie du genre: la culpabilité du «temps volé » à la famille ou des contraintes supplémentaires imposées au conjoint constitue une «spécificité» largement féminine (Jarty, 2009) produite par l'assignation sexuée de la prise en charge de la vie domestique. Mais le cas des chercheurs révèle un facteur pouvant alourdir ce sentiment de culpabilité: la flexibilité et l'autonomie dans l'organisation du travail qui permettent de décider du lieu et du moment de travail. Dans un contexte d'incertitude d'emploi, de régulation temporelle du travail faible et d'une injonction sociale à la prise en charge de la vie domestique par les femmes (retraduite quotidiennement dans les relations de travail), ces deux aspects socialement définis comme fortement favorables à une «conciliation» (Trancart et al., 2009), peuvent alourdir, chez les femmes, les risques d'être exposées à des rappels à l'ordre domestique et donc, éventuellement, à une forme de tiraillement. Autrement dit, la flexibilité est une chance pour de nombreux chercheurs qui insistent sur un «privilège » unique, mais elle peut conduire à être identifié d'emblée et/ou à s'identifier à une figure du carer pouvant être, s'il le faut, toujours présent au bon moment (Bessin et Gaudart, 2009).

On trouve ici un élément d'explication permettant d'éclairer le phénomène bien connu des «pères gagnants» (De Singly, 1994; Gadéa et Marry, 2000) (la venue d'un enfant produisant un effet sur la carrière plus favorable chez les hommes que chez les femmes). Si nos résultats de recherche ne peuvent pas prédire un tel effet sur la réussite ou l'échec à l'obtention d'un emploi stable dans la recherche, en revanche, nous avons pu observer lors de l'enquête exploratoire que les pères sont plus optimistes quant à la probabilité estimée d'obtenir une titularisation (moyenne de $62 \%$ ) que les mères (moyenne de $42 \%$ ) (voir Tableau 1). Un affinement de ces résultats afin d'analyser les différences de variances entre les groupes de chercheurs en fonction de leur statut conjugal (en couple/célibataire) et parental (avec/sans enfant) (test ANOVA) souligne qu'afin de maximiser subjectivement ses chances d'obtenir un poste définitif, il paraît préférable d'être célibataire pour une femme, et en couple et surtout père pour les hommes.

hommes seraient, moins qu'hier, insensibles aux «gratifications» de la parentalité, attachement qui pouvait (et peut toujours) apparaître «culturellement problématique pour les hommes dont il questionne beaucoup plus fortement l'identité» (Zarca, 2006: 58). 


\subsection{L'interférence travail-famille dramatisée par la trajectoire sociale}

Qualitativement, notre étude révèle une autre dimension encore peu appréhendée, bien qu'elle soit au cœur des approches intersectionnelles, qui, si elle ne peut être généralisée à partir de notre enquête, n'en reste pas moins significative: chez plusieurs mères rencontrées, issues de milieux populaires, le coût de la parentalité apparaît décuplé car l'accès à un poste de chercheur représente une promotion sociale hautement désirée à la fois parce qu'elle est valorisée par leur milieu d'origine et parce qu'elle marque simultanément une distance avec la domination masculine qui, estiment-elles, caractérise ce milieu. Le cas de Manuella a déjà été évoqué mais nous voudrions ici placer le zoom de l'analyse sur celui de Sia qui exprime de façon très critique des enjeux sociaux majeurs peu pris en compte dans la gestion des carrières scientifiques à l'université. Il est particulièrement suggestif d'un entremêlement du genre et de l'origine sociale dans l'interférence travail-famille producteur d'une profonde culpabilité. Cette chercheure présente une configuration familiale déficitaire sur le plan des ressources organisationnelles, ses parents et beaux-parents étant inaccessibles et son conjoint peu disponible (il travaille comme enseignant-chercheur dans une université éloignée de leur domicile). Elle a été socialisée dans un milieu qu'elle décrit comme modeste (son père était douanier et sa mère secrétaire-comptable) et qui, selon elle, écarte les jeunes filles de la voie des études longues et de la carrière professionnelle. Lorsqu'elle revient sur son parcours (ses deux thèses et ses séjours prolongés aux États-Unis), elle loue cette indépendance et ce mauvais tour joué aux déterminismes sociaux. À ses yeux, la carrière de chercheure représente une prise de distance par rapport à un milieu social perçu comme infériorisant les femmes. Le contenu de son travail, intellectuel, dont les contours échappent souvent à ses parents, lui procure une intense satisfaction et la contraint à une charge de travail élevée pour prouver à son entourage qu'elle travaille «réellement»: «Tu vois, par rapport à ma famille, fallait que je justifie ça [la recherche en littérature], pour eux c'est complètement loufoque [...].»

Cette proximité avec l'image du chercheur totalement engagé, forgée dans une trajectoire d'ascension (voire d'émancipation d'après les termes utilisés par Sia) sociale l'amène à considérer en premier lieu la parentalité comme inappropriée. Elle est inappropriée car le milieu scientifique ne s'y prête pas et car des enfants la contraindraient à mettre entre parenthèses sa carrière et à se rapprocher de son milieu social d'origine, de ses sœurs notamment, qui n'ont fait «aucune étude, elles se sont mariées très jeunes et ont eu des enfants très jeunes», un «modèle» qu'elle a «repoussé» tout en estimant qu'elle ne pourrait pas «vivre une vie sans enfants».

Sa volonté de toujours s'engager pleinement dans la carrière scientifique tient aussi au refus de se soumettre à la carrière de son mari avec lequel elle vit depuis quinze ans et qu'elle a accompagné aux États-Unis. Pour elle, ces affranchissements (du milieu d'origine et de la dépendance envers la situation professionnelle de son mari) expliquent une volonté de ne pas gâcher sa carrière en faisant des enfants. Toutefois, peu avant 40 ans, son désir d'enfants s'intensifie. Elle rentre alors en conflit 
avec son mari qui conditionne longtemps le projet d'enfant à l'obtention de positions professionnelles stables. Lorsqu'il obtient un poste comme chercheur, il attend que Sia obtienne un poste avant de faire un enfant. Il ajoute même comme condition celle de disposer d'une habitation pouvant le recevoir. La rencontre de plusieurs échecs professionnels (des candidatures comme maître de conférences en France pour lesquelles elle est fréquemment classée seconde) et de l'impossibilité d'avoir un enfant produit chez elle une longue période de déprime:

Les trois années jusqu'à ce que j'obtienne mon poste ici au FNRS, de chargée de recherches, j'suis vraiment passée par une période de... de dépression intense en fait. Parce que, ben voilà, $j$ 'approchais de la quarantaine, j'avais effectivement deux thèses, mais euh, je n'avais pas d'enfant. Or, ça devenait pour moi quelque chose qui était très important. Et je n'avais pas de poste définitif. [...] C'était hyper destructeur, parce qu'en fait j'me suis culpabilisée d'pas trouver d'travail, d'être toujours dans cette position d'deuxième, si tu veux.

Sa détresse est dès lors atténuée lorsque son mari accepte de s'engager dans un projet parental. Leur enfant constitue l'issue d'un projet ancien, douloureux dans lequel Sia s'investit avec ardeur et envers lequel ses attentes sont accrues par les possibilités offertes, selon elle, par la flexibilité et la disponibilité de son travail. Cohabitent ainsi chez Sia une forte aspiration à la parentalité, produite par une trajectoire familiale à la fois contrariée et conditionnée par l'obtention d'un poste stable, et une forte aspiration au travail forgée dans une trajectoire sociale ascendante, témoignant d'une volonté de quitter un milieu social synonyme, à ses yeux, de subordination féminine. L'intrication élevée du privé et du professionnel autour du projet d'enfant fabrique un rapport dramatisé à la fois au travail, à l'emploi et à la famille, fait d'attentes élevées. Les défauts de sa configuration familiale la positionnent en première ligne dans la gestion de la vie domestique, dont celle touchant à son fils. Certes, cela lui procure de grandes satisfactions. Mais, d'une part, cela la rapproche d'une figure maternante potentiellement infériorisante, selon elle, qu'elle s'est employée à évacuer. D’autre part, comme elle entend donner le change au monde de la recherche afin de valider entièrement une trajectoire d'ascension sociale jusqu'ici, à ses yeux, inachevée, la double demande professionnelle et parentale conduit à une culpabilité qui se porte à la fois sur le rapport à son enfant et le rapport à son activité professionnelle. Devant l'indisponibilité de son mari, l'absence de ressources familiales permettant de prendre en charge leur enfant, le retour du congé maternité provoque chez Sia une intense culpabilité. Lorsqu'elle doit ainsi laisser son fils âgé de trois mois à la crèche, un bon moyen pour elle de ne pas être «trop maternante», elle se plaint de ne pas parvenir à être une «bonne mère», souvenirs qu'elle exprime en pleurant lors de l'entretien.

L'interférence entre vie familiale et travail prend donc chez elle les formes d'une perturbation importante, majorée par une trajectoire conjugale et sociale singulière. Ces facteurs produisent ensemble une tension, un durcissement dans le rapport au travail, qui du fait de la hausse de coûts d'accès à la carrière scientifique, s'abîme: la question de la justice est mise en avant pour dénoncer un milieu scientifique qui rétribue peu les efforts consentis (relus progressivement sous la forme de sacrifices). 


\section{CONCLUSION}

L'interdépendance des sphères professionnelle et familiale a été étudiée dans de nombreux travaux sur l'«articulation» ou la «conciliation». Ces travaux l'appréhendent sous l'angle de l'emploi et du genre, sous l'angle organisationnel (les entreprises) ou institutionnel (les politiques publiques). Partant de l'expérience du travail au sein d'un groupe professionnel circonscrit (les chercheurs postdoctorants), et couplant une analyse des interdépendances objectives à celle des interstructurations subjectives, la thématisation que nous proposons en termes d'interférence amène à porter un regard renouvelé sur ce phénomène.

Cet article permet alors de percevoir combien l'analyse du rapport que les chercheurs entretiennent avec leur travail peut difficilement faire l'économie d'une prise en compte de leur situation privée, et familiale en particulier (ce qui est généralisable à d'autres groupes professionnels). Il ne contredit pas les enseignements des recherches antérieures sur les carrières professionnelles, mais il en précise et complexifie certains résultats. Ainsi, il éclaire une réalité encore insuffisamment explorée: celle des interactions des hommes avec leur vie familiale. On comprend précisément ici pourquoi les pères (mais pas tous) peuvent être «gagnants», y compris subjectivement, souvent plus que les mères, avec la parentalité. Simultanément, on comprend mieux que ce n'est pas uniquement le fait d'être une femme ou une mère qui crée une situation perturbatrice mais bien un type d'interférence entre la configuration familiale et la configuration professionnelle. En outre, le cas de Sia que nous avons développé indique combien une approche intersectionnelle faisant intervenir l'origine sociale affine l'analyse.

Par conséquent, l'entrée analytique développée dans le cadre de cet article participe au repérage des mécanismes et processus invisibles de discriminations que la régulation des universités produit. Le genre, la situation familiale et la trajectoire sociale apparaissent ensemble comme un «filtre caché» dans la gestion des carrières, caché car les critères consacrés à la mesure de «l'excellence scientifique» sont conçus à la fois comme neutres sexuellement et socialement, et désindexés des conditions de vie des chercheurs. Ce que l'analyse sociologique tend à déconstruire.

\section{RÉSUMÉ}

À partir de l'analyse d'entretiens menés auprès de jeunes chercheurs-parents, cet article met en avant les effets différenciés de la vie familiale sur la manière dont les individus voient et font leur travail. Il étudie le rapport au travail des chercheurs comme le produit de la rencontre entre une configuration familiale (ressources matérielles à disposition en vue de l'organisation quotidienne, attentes envers la vie familiale, la trajectoire familiale) et une configuration professionnelle (ressources matérielles et symboliques conférées par le milieu professionnel, trajectoires professionnelles observées par les individus, regard sur le métier et ses chances d'y faire carrière). En soulignant le phénomène d'interférence travail/famille, l'article invite à articuler l'analyse des interdépendances objectives entre ces deux milieux de vie à l'analyse de leurs interstructurations subjectives.

Mots clés: rapport au travail, articulation vie professionnelle/vie privée, travail scientifique, parentalité 


\begin{abstract}
Based on the analysis of interviews with young researcher-parents, this article presents the differentiated effects of family life on the way individuals see and carry out their work. It studies how researchers consider work as the product of the meeting between a family configuration (material resources that are available for the organization of daily life, expectations regarding family life, family history) and a professional configuration (material and symbolic resources provided by the professional environment, professional history observed by individuals, perspective on the profession and chances of having a professional career). Stressing work/ family interferences, the article encourages the articulation of the analysis of the objective interdependencies between these two living environments with the analysis of their subjective interstructurations.
\end{abstract}

Key words: Attitude to work, articulation of professional life/private life, scientific work, parenting.

\title{
RESUMEN
}

A partir del análisis de entrevistas realizadas con jóvenes investigadores y padres a la vez, este artículo subraya los efectos diferenciada de la vida familiar en canto a la manera como los individuos ven y realizan su trabajo. Se estudia la relación de los investigadores con el trabajo, como producto del encuentro entre una configuración familiar (recursos materiales a disposición con miras a la organización cotidiana, acuerdos con la vida familiar, trayectoria familiar) y una configuración profesional (recursos materiales y simbólicos conferidos por el medio profesional, trayectorias profesionales observadas por los individuos, percepción acerca del oficio y sus posibilidades de carrera). El artículo subraya el fenómeno de interferencia trabajo/familia y propone articular el análisis de las interdependencias objetivas entre estos dos medios de vida con el análisis de sus interestructuraciones subjetivas.

Palabras clave: relación con el trabajo; articulación vida profesional/vida privada, trabajo científico; paternidad.

\section{BIBLIOGRAPHIE}

Ait, A. et N. Rouch (2013), «Le «je suis débordé» de l’enseignant-chercheur», Temporalités, n 18. [En ligne: http://temporalites.revues.org/2632]

ANEF (2014), Le genre dans l'enseignement supérieur et la recherche, Paris, La Dispute.

Balibar, S. (2014), Chercheur au quotidien, Paris, Le Seuil.

Barrrere-Maurisson, M.-A. (2003), Travail, famille: le nouveau contrat, Paris, Gallimard.

Barrrere-Maurisson, M-A. et D.-G. Tremblay (dir.) (2009), Concilier travail et famille. Le rôle des acteurs. Québec-France, Presses de l'Université du Québec.

Beaufays, S. et B. Krais (2005), «Femmes dans les carrières scientifiques en Allemagne: les mécanismes cachés du pouvoir», Travail Genre et Sociétés, vol. 14, n² 2, p. 49-68.

Beld, D. (1973), The Coming of Post-Industrial Society: A Venture in Social Forecasting, New York, Basic Books.

Benschop, Y. et M. Brouns (2003), «Crumbling Ivory Towers: Academic Organizing and its Gender Effects», Gender Work and Organization, vol. 10, n 2, p. 194-212.

Bessin, M. et C. Gaudart (2009), «Les temps sexués de l’activité: la temporalité au principe du genre?», Temporalités, nº 9. [En ligne: http://temporalites.revues.org/979] 
Blair-Loy, M. (2003), Competing Devotions. Harvard University Press.

Buscatto, M. et C. Marry (2009), «Le plafond de verre dans tous ses éclats. La féminisation des professions supérieures au $\mathrm{xx}^{\mathrm{e}}$ siècle, Introduction du numéro spécial», Sociologie du travail, vol. 51, $\mathrm{n}^{\circ} 2$, p. $170-182$.

Cacouault-Bitaud (2007), Professeurs... mais femmes. Carrières et vies privées des enseignantes du secondaire au $\mathrm{XX}^{e}$ siècle, Paris, La Découverte.

Case, S. et B. Richley (2013) «Gendered institutional research cultures in science: The post-doc transition for women scientists», Community, Work and Family, 16 (3) p. 327-349.

Collectif (1984), Le sexe du travail, Grenoble, PUF.

Coser, L. (1974), Greedy Institutions, New York, The Free Press.

Currie, J., P. Harris et B. Thiele (2000), «Sacrifices in Greedy Universities: Are they gendered?», Gender and Education, $\mathrm{n}^{\circ} 12$, p. 269-291.

Dany, F., S. Louvel et A. Valette (2011), «Academic Careers: The limits of the "boundaryless approach" and the power of promotion scripts», Human Relations, n 64, p. 971-996.

Datchary, C. (2008), «Gérer la dispersion: un travail collectif», Sociologie du travail, vol. 50, p. 396-416.

De singly, F. (1994), Fortune et infortune de la femme mariée, Paris, PUF.

De singly, F. (1996), Le soi, le couple, la famille, Paris, Nathan.

Del Rio Carral, M. et B. Fusulier (2013), «Jeunes chercheurs face aux exigences de disponibilité temporelle», Temporalités, n 18 [En ligne: http://temporalites.revues.org/2614]

Devreux, A.-M. (1985), «Les différentes étapes de la déconstruction — reconstruction des objets et des concepts: trois exemples ", Cahiers de l'APRE, n 3, CSU-CNRS, p. 7-28.

Etzkowitz H., Kemelgor C. et Uzzi B. (2000), Athena unbound: The advancement of women in science and technology, Cambridge University Press.

Fagnani, J. et M.-T. Letablier (2001), Familles et travail: contraintes et arbitrages, Paris, La documentation Française.

Ferrand, M. (1984), «Paternité et vie professionnelle», in (coll.) Le sexe du travail, Grenoble, PUG, p. 127 139.

Fusulier, B. (dir.) (2003), «Articuler travail et famille», Les Politiques Sociales, n 3-4.

Fusulier, B. (2011a), Articuler vie professionnelle et vie familiale. Étude de trois groupes professionnels: les infirmières, les policiers et les assistants sociaux, Louvain-la-Neuve, Presses universitaires de Louvain.

Fusulier, B. (2011b), «Le concept d'ethos professionnel», Recherches sociologiques et anthropologiques, vol. 42, n 1. [En ligne: http://rsa.revues.org/661]

Fusulier, B. et M. Del Rio Carral (2012), Chercheur-e-s sous haute tension!, Louvain-la-Neuve, Presses de l'Université de Louvain.

FusulieR, B. et D.-G. Tremblay (dir.) (2013), «Articulation travail/famille: une entrée par les groupes professionnels», Revue internationale Enfances, Familles, Générations, vol. 18, nº 18.

Gadea, C. et C. Marry (2000), «Les pères qui gagnent: descendance et réussite professionnelle des ingénieurs », Travail, Genre et Sociétés, n 3, p. 109-135.

Hantrais, L. et M.-T. Letablier (1995), La relation famille-emploi: Une comparaison des modes d'ajustement en Europe, Centre d'études de l'emploi, dossier $n^{\circ} 6$.

Hendrickson, R., M. Mueller et J. Strand (2011), «Policital Science Careers at Comprehensive Universities: Building Careers at “Greedy” Institutions », Political Science \& Politics, n 44, p. 129-134.

Hochschild, A. (1997), The Time Bind, Henry Holt and Company.

JARTY, J. (2009), «Les usages de la flexibilité temporelle chez les enseignantes du secondaire», Temporalités, $\mathrm{n}^{\circ}$ 9. [En ligne: http://temporalites.revues.org/1057]

Kanter, R. M. (1977), Work and Family in the United States: A Critical Review and Agenda for Research and Policy, New York, Russel Sage Fundation.

Lapeyre, N. (2004), «Les femmes architectes: entre créativité et gestion de la quotidienneté», Empan, $\mathrm{n}^{\circ} 53$, p. $48-55$.

Lapeyre, N. (2008), «De l'inflexion des normes temporelles à la redéfinition de "l'éthos professionnel”. L'exemple des femmes dans les professions libérales en France», in Le Bihan, B. et C. Martin (dir.), Concilier vie familiale et vie professionnelle en Europe, Rennes, Éditions de l'ENSP, p. 215-228. 
Le Bihan-youinou, B. et C. Martin (dir.) (2008), Concilier vie professionnelle et vie familiale en Europe, Rennes, Presses de l'EHESP.

Lefeuvre, N. et N. Lapeyre (2013), «L'analyse de l'articulation des temps de vie au sein de la profession médicale en France: révélateur ou miroir grossissant des spécificités sexuées», Enfances, Familles, Générations, nº 18, p. 72-93.

Marry, C. (2004), Les femmes ingénieurs. Une révolution respectueuse, Paris, Belin.

Marry, C. et I. JONAS (2005), «Chercheuses entre deux passions», Travail, Gsenre et Sociétés, vol. 14, n², p. 69-88.

Meda, D. (2001), Le temps des femmes. Pour un nouveau partage des rôles, Paris, Flammarion.

Merton, R. (1969), «The Matthew Effet», Science, 159 (3810), p. 56-63.

Meulders, D., O'dorchai, S. et Simeu, N. (2012), Les inégalités entre femmes et hommes dans les universités francophones de Belgique, Université Libre de Bruxelles, rapport de recherche GENIUF.

Musselin, C. et F. Pigeyre (2008), «Les effets des mécanismes du recrutement collégial sur la discrimination: le cas des recrutements universitaires», Sociologie du travail, vol. 50, p. 48-70.

Nicole-Drancourt, C. (2009), Conciliation travail-famille: attention travaux, Paris, L'Harmattan.

Pailhe, A. et A. Solaz (2009), Entre travail et famille, Paris, La Découverte.

Perivier, H. et R. Silvera (2010), «Maudite conciliation», Travail, genre et sociétés, n 24, p. 25-27.

Pigeyre, F. et M. SAbatier (2011), «Les carrières des femmes à l'université: une synthèse de résultats de recherche dans trois disciplines", Politiques et Management Public, vol. 28, $\mathrm{n}^{\circ} 2$. [En ligne]

Rossiter, M. (1993), «The Matthew Matilda Effect in Science», Social Studies of Science, 23 (2), p. 325-341.

SARfati, F. (2012), Du côté des vainqueurs. Une sociologie de l'incertitude sur les marchés du travail, Septentrion, Lille.

She figures (2013), Gender in Research and Innovation, Publications Office of the European Union, Luxembourg.

Trancart, D., N. GEORGES et D. MEDA (2009), "Horaires de travail des couples, satisfaction et conciliation entre vie professionnelle et vie familiale», in PaIlhe, A. et A. Solaz (dir.), Entre travail et famille, Paris, La Découverte, p. 55-78.

Tremblay, D.-G. (2004), Conciliation emploi-famille et temps sociaux, Québec/Toulouse, Téluq/Octares.

Tremblay, D.-G. (dir.) (2005), De la conciliation emploi-famille à une politique des temps sociaux, Québec, Presses de l'Université du Québec.

Tremblay, D.-G. (2012), Articuler emploi et famille: le rôle du soutien organisationnel au cour de trois professions (infirmières, travailleuses sociales et policiers), Presses de l'Université du Québec.

Zarca, B. (2006), "Mathématicien: une profession inégalitaire et masculine», Sociétés Contemporaines, $\mathrm{n}^{\circ} 64, \mathrm{p} .41-65$. 


\section{AN NEXE}

Tableau 1: Estimation de la probabilité (en \%) d'obtenir un poste permanent selon le statut parental et le sexe

\begin{tabular}{l} 
Si vous visez un poste permanent dans la recherche universitaire, à quel pourcentage estimez-vous la \\
probabilité de concrétiser cet objectif professionnel? \\
\hline
\end{tabular}

Source: Questionnaire auprès des chargés de recherches du FNRS réalisé en 2010 et 2011. 